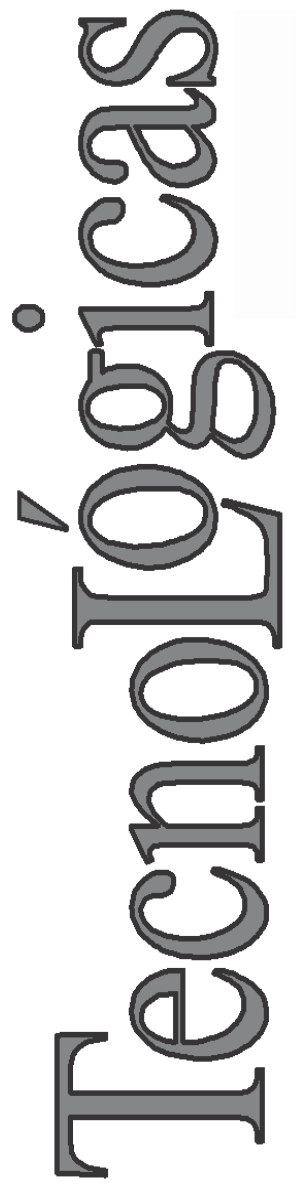

\title{
Modelo de Calidad de Servicio a Nivel de Enlace de Datos para Tráfico de Misión Crítica sobre Redes IEEE 802.11g en Modo Infraestructura
}

Quality of Service Model on Data Link Layer for Mission Critical Traffic on IEEE 802.11g Networks in Infrastructure Mode

Gerald B. Fuenmayor-Rivadeneira ${ }^{1}$

1 Grupo de investigación INVTEL, Facultad de Ingeniería de Telecomunicaciones, División de Ingenierías, Universidad Santo Tomás, Bogotá-Colombia geraldfuenmayor@usantotomas.edu.co 


\title{
Resumen
}

Este artículo presenta en forma sintetizada una revisión del estado del arte del estudio de la Calidad de Servicio para tráfico de misión crítica en redes de área local inalámbricas que utilizan el protocolo IEEE 802.11g. Esto con el fin de resaltar investigaciones previas que por su aporte se constituyan en un referente para orientar la propuesta de una nueva forma de abordar el aseguramiento de la calidad de servicio para este tipo de tráfico utilizando el protocolo mencionado. La revisión se basa en artículos académicos y empresariales elaborados durante el presente lustro. Como resultado de dicha revisión se evidencia que han sido muchos los esfuerzos para abordar el tema pero aún existen vacíos en la caracterización del tráfico de misión crítica y la garantía de la calidad del servicio para el mismo, debido a las nuevas aplicaciones informáticas y a la gran acogida de las redes WiFi en el ámbito empresarial y gubernamental; lo que ha generado un aumento en la demanda de los canales de acceso y, por ende, un desafío para los avances ya conocidos en la materia, tal como IEEE 802.1q.

\section{Palabras clave}

Telecomunicaciones; calidad de servicio; WiFi; misión crítica; simulación.

\begin{abstract}
This article presents a synthesized review as state of the art of the study of QoS for mission-critical traffic in wireless local area networks that use the IEEE $802.11 \mathrm{~g}$ protocol. This is to highlight previous research for their contribution will constitute a reference to guide a proposed new approach to ensuring the quality of service for this type of traffic using the above protocol. The review is based on academic and business items made during the current five years. As a result of this review it is evident that there have been many efforts to address the issue but there are still gaps in the characterization of mission-critical traffic and ensuring quality of service for the same, due the new applications and the large host of WiFi networks in business and government, which has led to increased demand for access channels and, therefore, a challenge to the progress already known, such as IEEE 802.1q.
\end{abstract}

\section{Keywords}

Telecommunications; quality of service; WiFi; mission critical; simulation. 


\section{INTRODUCCIÓN}

Actualmente en las corporaciones es cada vez más común encontrar redes de área local inlámbrica (Local Area Network) que se han extendido mediante componentes inalámbricos, específicamente utilizando la tecnología IEEE $802.11 \mathrm{~g} / \mathrm{n}$ ( WiFi). De la misma manera, la apropiación de las herramientas informáticas y de comunicaciones han permeado todas las áreas organizacionales, al punto que le han confiado el transporte de paquetes que involucran información crítica para un número indeterminado de procesos; por ello, es menester que los encargados de velar por la confiabilidad de la transmisión cuenten con un marco de referencia que le ayuden a la parametrización de los niveles de calidad de servicio requeridos por las aplicaciones. En este sentido, muchos estudios se han realizado para establecer la calidad de servicio en diversas tecnologías de redes; sin embargo, es necesario conocer de qué manera el tráfico ve afectado sus condiciones de calidad de servicio al pasar entre hosts interconectados por una estación base en una red IEEE $802.211 \mathrm{~g}$, so pena de una pobre cualificación del mismo y posibles degradaciones no previstas de paquetes o tramas que pudieran perjudicar los servicios que soportan. El estudio del protocolo precitado se debe a su amplia adopción por la industria y la disponibilidad de módulos de simulación para su análisis, lo cual sería un punto inicial para abordar la tecnología IEEE $802.11 \mathrm{n}$ en un estudio posterior.

Adicional a lo precedente, se debe tener en cuenta algunas connotaciones que circundan el estudio de calidad de servicio en redes LAN inalámbricas. En este sentido, no solo se deben abordar las capas superiores sino que al igual se deben incluir los factores atenuantes del ambiente que hacen necesario tener en cuenta la capa física. Al respecto, (Panousopoulou, 2004) menciona que el comportamiento de un medio inalámbrico tiene un impacto significativo en la transmisión, lo cual indica la pertinencia de tener en cuenta estudios que modelen interferencias en el medio e incluso variación de la potencia de transmisión, siendo este último un factor de calidad que también entra en juego y se debe tener en cuenta. Dado lo anterior, se plantean los siguientes siguiente cuestionamientos: ¿De qué manera el tráfico de un servicio de 
misión crítica ve afectado sus condiciones de calidad de servicio al nivel de enlace de datos al pasar entre un host y otro en una red WiFi IEEE 802.11g en modo infraestructura, sobre todo cuando aumenta el número de hosts conectados a la estación base dentro del rango de distancia que impone la recomendación?¿Cómo se podría mejorar el nivel de calidad de servicio para dicho tráfico en las condiciones mencionadas?

Para dar respuesta a lo anterior es imperativo conocer el desarrollo investigativo alrededor de la temática que sustente una investigación, de la cual se espere arrojar un aporte significativo a quienes ponen sus datos más sensibles sobre una red de las características precitadas. De hecho, este cuestionamiento se ve alimentado por artículos como el editado por (Graeme Bell, 2007), en donde se expresa la siguiente pregunta: "iSo would I consider running a mission-critical process control system on an $802.11 \mathrm{bg}$ wireless network?". Bell, si bien muestra una alternativa para poder afrontar este cuestionamiento de manera positiva, deja entrever que el tema reviste atención.

\section{METODOLOGía}

Para este artículo se consultaron trabajos realizados desde el año 2003 hasta la fecha. No obstante, la concentración de artículos estudiados se ubica entre el año 2008 y 2013, es decir los últimos cinco años. Se tuvieron en cuenta solo los artículos o textos que trataron el tema de calidad de servicio, especialmente en entornos inalámbricos WLAN o similares, como también trabajos que revisten interés por las herramientas de análisis o simulación utilizadas.

\section{RESULTADOS Y DISCUSIÓN}

\subsection{Hacia un Estado del Arte}

Hoy es visible el hecho de que muchas organizaciones han encontrado en las redes de telecomunicaciones una herramienta 
invaluable para transportar información que en muchos casos obedecen a aplicaciones en tiempo real (telecontrol, por ejemplo). Muchos procesos de negocios también utilizan las redes para el soporte de aplicaciones de gestión que involucran información de misión crítica para la empresa; tal es el caso del proceso de facturación en una central de conmutación la cual es gestionada frecuentemente desde un lugar distinto a su ubicación física. Muchos ejemplos se pueden mencionar, pero en este caso lo que realmente interesa es como hacer que a un determinado tipo de tráfico se le dé la prioridad necesaria en la red para que el servicio que lo genera no se afecte en su desempeño; lograr este cometido es lo que implica hablar de calidad de servicio ( $Q o S$ por su sigla en inglés).

La calidad de servicio puede considerarse una herramienta para la seguridad (Szigeti, 2004; Kisner, 2010), ver Fig. 1, lo cual no es extraño si se tiene en cuenta un contexto en donde un sistema de misión crítica supedita su eficacia a los datos recibidos; si estos últimos llegan con retardos o mutilados se puede correr el riesgo de respuestas demoradas o inapropiadas del sistema. (Szigeti, 2004) realiza una conceptualización sobre el tema de calidad de servicio, destacando su evolución y las particularidades que ofrecen distintos tipos de tráfico frente al mismo.

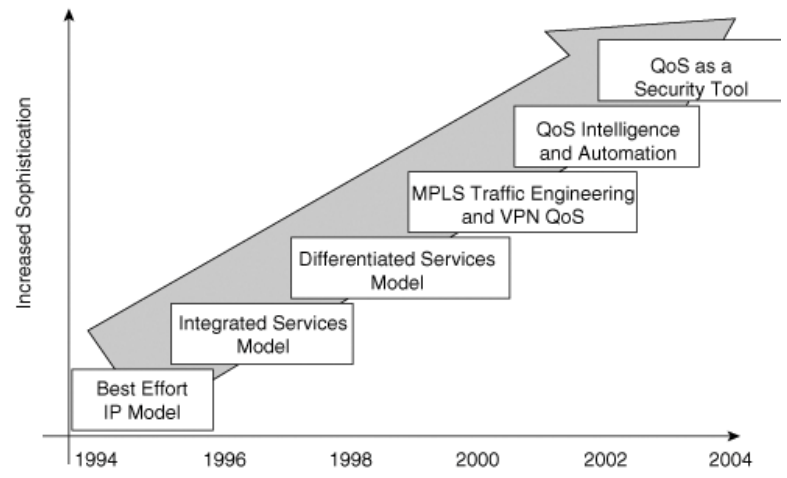

Fig. 1. Evolución de QoS. Fuente: Szigeti, 2004

(Serrano, 2006) en su tesis doctoral muestra como el estándar IEEE 802.11e, el cual a finales del año 2005 involucró aspectos para el manejo de QoS en WiFi, presenta ciertas deficiencias y 
deja entrever el hecho de que aún falta desarrollar más investigación que conduzca a mejorar las prestaciones en QoS de este tipo de red. En este mismo sentido, (Tao, 2004) en su artículo señala que la eficacia de la función mejorada de acceso al canal (enhanced distributed channel access - EDCA) introducida en IEEE 802.11e, aún no ha sido fuertemente investigada.

Por su parte (Prasad, 2004) desarrolla en su libro toda una temática frente a la aplicación de calidad de servicio en redes WLAN destacando algunos aspectos en lo que se refiere a seguridad. (Kweon \& Shin, 2003) trabajaron en el modelamiento de tráfico estadístico de tiempo real, en donde se estudian algunos aspectos paramétricos que condicionan el nivel de calidad de servicio de este tipo de tráfico en una red Fast Ethernet. Ellos ofrecieron un punto de referencia importante para establecer los parámetros de calidad a estudiar y una forma para abordar la caracterización del tráfico de misión crítica.

En lo que al uso de simuladores respecta, (Flórez, et al., 2006) realizaron una comparación a nivel de paquetes de tres simuladores de red, los cuales son: NS2, Modeler de OPNET y NCTUns. Esta comparación ofrece un punto de referencia importante para la selección de un simulador de red apropiado para estudiar el tráfico de misión crítica. (Franceschinis, et al., 2005) proporcionaron información valiosa para abordar las mediciones de los paquetes a nivel TCP en una transmisión vía inalámbrica. Por su parte, (Sen, 1998) abordó el tema de degradación de calidad de servicio desde la perspectiva de redes inalámbricas incluso con tráfico de tiempo real, lo cual ofrece en principio una excelente referencia para la caracterización del tráfico de misión crítica aunque se limita a redes multimedia de tercera generación.

Angeja, et al., (2004) propusieron un modelo estadístico para la llegada y pérdida de paquetes en una red WLAN IEEE 802.11g que trabaja en modo infraestructura. Este trabajo ofrece una referencia importante para lo que tiene que ver con la caracterización del tráfico de misión crítica y la posibilidad de llegar a un modelo estadístico del mismo. A nivel de patentes, (Malaney, 2006) inventó un aparato que desarrolla mapas de predicción con respecto a algún parámetro de calidad de servicio a nivel de una red inalámbrica, lo cual constituye un aporte a la temática subyacente. 
Bernal \& Salcedo, (2004) abordaron el tema de calidad de servicio a nivel de red inalámbrica, aunque orientado a celulares más no a redes LAN, no obstante ofrecen una alternativa para abordar el proceso de evaluación de la calidad de servicio. Por otro lado, (Báez, 2001) aborda el tema de calidad de servicio en redes multiservicio, lo cual marca una referencia importante debido a la caracterización del tráfico en servicios de datos. Yang, (2001) referencia algunos parámetros de calidad citando su respectivo RFC (Request for conment), lo cual es una utilidad a la hora de especificar tráfico.

Una mirada más reciente permite presentar trabajos que dan constancia de las falencias de IEEE 802.11e y proponen ajustes en la subcapa de acceso al medio para contrarrestar efectos del retardo y mejorar la calidad de servicio para tráfico de aplicaciones multimedia de tiempo real. Para esto se aplicó un modelo analítico (Zhao, 2012). (Yang, et al., 2012) desarrollaron un modelo estocástico con redes de Petri para ofrecer calidad de servicio en redes personales móviles inalámbricas multi salto. Esto se hizo para mejorar los niveles de calidad de servicio para el tráfico de videoconferencia y electrocardiografía que se transmiten a través de la red.

La aplicación de WLAN para la comunicación entre vehículos que viajan en carretera también ha sido un problema abordado en donde se presentan las falencias que se deben resolver para hacer más viable este escenario y se hace una revisión de la tecnología existente para ello. En este escenario se contempla el acceso del vehículo a la WLAN a través de puntos de accesos de carretera (Hasan, et al., 2012). Otros trabajos que guardan relación con este también han sido estudiados (Rappaport, et al., 2011; Simo 2010). Por otro lado, (Ullah, 2012) en su tesis doctoral realiza un análisis comparativo que muestra los costos, bondades y debilidades de distintas tecnologías inalámbricas como entre las que se encuentran Long Term Evolution (LTE) e IEEE 802.11. Este análisis resalta una serie de resultados útiles como criterio para selección de este tipo de tecnología para ser utilizada en redes públicas. (Kritzinger, et al., 2010; Navajas, et al., 2005) trataron problemáticas similares. 
Un trabajo de (Al-Madani, et al., 2012) muestra un estudio de transmisión de video mediante un sistema middleware de distribución de datos a través de IEEE $802.11 \mathrm{~g}$, en el que se evaluaron parámetros de desempeño de la transmisión y se investigó la calidad de servicio para video streamming. La calidad de experiencia también ha sido tenido en cuenta en esta revisión, con el trabajo de (Cano \& Cerdan, 2012) en donde a partir del análisis de un servicio VoIP sobre WLAN, evalúan la calidad de servicio (QoS) y de experiencia (QoE). Ellos concluyeron que la evaluación QoE no coincide con QoS debido a las disparidades entre sus criterios de evaluación. Igualmente resaltan el hecho de que la popularidad de la aplicación incide sobre su QoE. Por su parte, una patente de (Wentink, 2012) aborda el problema del aumento de las cabeceras de las tramas de inicialización (beacon) almacenadas en las memorias de los puntos de acceso (Access Point) inalámbricos en una WLAN. EL $A P$ debe suspender su standby para revisar sus buffers periódicamente. La patente muestra una técnica que reduce el tiempo de consulta de este tipo de tramas.

Una investigación de (Hu, et al., 2011) presenta un modelo de QoS asimétrico evaluado de manera experimental en donde se muestra que debido al parcial despliegue que ha tenido la tecnología IEEE 802.11e, es necesario mejorar las condiciones de QoS en clientes que no admitan dicha tecnología. Los resultados muestran un buen rendimiento, incluso en escenarios de congestión y con tráfico sensible al retardo como VoIP. Por su parte, (Mitra, et al., 2011) revela un método teórico que intenta mejorar el rendimiento del acceso de estaciones móviles en una WLAN, para lo cual aplica un algoritmo asociado a la teoría de juegos en donde encontraron distintos resultados de rendimiento entre IEEE 802.11b e IEEE 802.11g. En este trabajo se aprecia que el problema de mejorar QoS aún está en desarrollo y que puede aportar al mismo problema en IEEE 802.11n.

Un trabajo de modelación analítica propuso recientemente (Deb \& Hartman, 2012) para la optimización de las ventanas de contención en IEEE 802.11e. El análisis matemático da como resultado un algoritmo que resuelve el modelo analítico modelando el estado de la red como una función de los parámetros de la MAC y desarrollando la solución de forma distribuida, siendo este deta- 
lle la principal contribución según los autores. Al final de las simulaciones se obtuvieron menor tasa de pérdidas de paquetes y de retrasos, además de una diferenciación de QoS.

(Sarkar \& Paolini, 2011) analizaron el comportamiento de IEEE 802.11 e y concluyeron que el tráfico de tiempo no real ve mermada su oportunidad para traficar por la red debido a las prioridades del tráfico de tiempo no real. Por esta razón, ellos propusieron un protocolo a nivel MAC (Medium Access Control) más justo en el que el tráfico de tiempo no real también tenga oportunidad y no "se muera de hambre". La simulación de comprobación se hizo con QualNet. Los resultados mostraron un acceso más justo a través de la mejora de los tiempos de turno. Por su lado, (Bhushan \& Ganguly, 2011) en su tesis propusieron una mejora a nivel MAC en WLAN donde variaron la ventana de contención también, de manera no uniforme buscando resultados similares a los de (Sarkar \& Paolini, 2011). También aplicaron QualNet.

En el ámbito médico se aprecia el interés de las WLAN ( $\mathrm{Pa}-$ quette, 2011; Vassis, 2010), un ejemplo más es el trabajo de (Paquette, 2011), el cual propone un laboratorio para prueba de conexión y uso de equipos médicos que utilizan la red inalámbrica. Con otra mejora aparece (Gaur, 2011), el cual aborda el problema de selección de canal que hacen los puntos de acceso y clientes de una WLAN. En este trabajo se desarrolló un módulo de selección de canal apoyado en QoS el cual se convierte en su principal aporte. De la misma manera trabajos como el de (Villegas, 2008) en que aborda el tema de canales, sobre todo a nivel de asignación de frecuencias con espectro compartido.

El tráfico de misión crítica también ha sido estudiando por (Nwizege, 2011; Cena, et al., 2009; Cena, et al., 2010). Con el trabajo de Nwizege se realizaron diversas simulaciones que dieron como conclusión que el control de aplicaciones de baja prioridad mejora la eficacia de las de alta prioridad. El estudio de WLAN también ha abordado aspectos del espectro y gestión de la energía e interferencias, en donde varios estudios han tratado de mejorar estas variables en beneficio de una mejor QoS, tal es el trabajo realizado por (Tinnirello \& Bianchi, 2010; Nieminen, et al., 2010; Aidogdu, 2010). 


\section{CONCLUSIONES}

Es fácil encontrar estudios de calidad de servicio a nivel WiFi, incluso estudios de degradación en la misma; lo que no es muy visible y fácil de encontrar es un estudio que aborde la degradación de la calidad de servicio cuando el tráfico correspondiente a un servicio de datos de misión crítica en este escenario. Por tanto es menester una investigación que llene este vacío. De manera complementaria se debería ofrecer una especificación para el tráfico de misión crítica, que cubra incluso el tráfico de las aplicaciones más modernas, como es el caso del control robótico a distancia o la esteganografía de datos.

\section{REFERENCIAS}

Al-Madani, B., Al-Roubaiey, A., \& Al-Shehari, T. (2012). Wireless video streaming over Data Distribution Service middleware. En Software Engineering and Service Science (ICSESS), IEEE 3rd International Conference on. IEEE, p. 263-266.

Angeja, J., Carvalho, L. \& Navarro, A. (2004). "802.11G WLAN modeling for real time packet communication", ;in Proc. PIMRC, 2958-2962.

Aydogdu, C. (2010). An Analytical Model of IEEE 802.11 DCF for MultiHop Wireless Networks and Its Application to Goodput and Energy Analysis. Tesis Doctoral. bIlkent university.

Báez Pérez, S. et al. (2001). Elementos y requerimientos para el diseño de una red multiservicio que involucre estrategias de calidad de servicio (QoS) y planteamiento de un ajuste a la red de la Universidad Nacional de Colombia basado en estas estrategias. Tesis. Dir. Edgar Chaparro. Universidad Nacional de Colombia. Bogotá, D. C.

Bernal B., C. A. (2004). Evaluación de la calidad del servicio (QoS) en una red inalámbrica $3 \mathrm{G}$ con arquitectura UMTS soportada en tecnología WCDMA, mediante el uso de software para simulación de redes. Tesis. Dir. Octavio José Salcedo Parra. Universidad Nacional de Colombia. Bogotá, D. C.

Bhushan, S. \& Ganguly, N. Study of QoS Management In IEEE 802.11 and 802.11 e MAC Layer Protocols. 2011. Tesis Doctoral.

Cano, M. D. \& Cerdan, F. (2012). Subjective QoE analysis of VoIP applications in a wireless campus environment. Telecommunication Systems, $49(1), 5-15$. 
Cena, G., Seno, L., Valenzano, A. \& Zunino, C. (2010). On the performance of IEEE 802.11 e wireless infrastructures for soft-real-time industrial applications. Industrial Informatics, IEEE Transactions on, 6(3), 425-437.

Cena, G., et al. (2009). Evaluation of real-time communication performance in QoS-enabled infrastructure WLANs. En Emerging Technologies \& Factory Automation, 2009. ETFA 2009. IEEE Conference on a. IEEE. 1-7.

Deb, B. \& Hartman, M. J. (2012). Distributed optimization of Contention Windows in 802.11 e MAC to provide QoS differentiation and maximize channel utilization. En World of Wireless, Mobile and Multimedia Networks (WoWMoM), 2012 IEEE International Symposium on a. IEEE, 1-7.

Dharanikota, S. (2005). QoS monitoring system and method for a highspeed DiffServ-capable network element. U.S. Patent No 6,914,883.

Flores L., G., Paredes F., M., Jammeh, E., Fleury. M. J. Reed, M. \& Ghanbari, M. (2006). Análisis a Nivel-Paquete de Simuladores de Red Contemporáneos. IEEE Latin America Transactions, 4(4).

Franceschinis, M., Mellia, M. M. \& Munafò, M. (2005). Measuring TCP over WiFi: A Real Case. Dipartimento di Elettronica - Politecnico di Torino - Italy.

Gaur, S. (2011). System and method of QOS-based channel selection for WLAN access points or stations. U.S. Patent No 7,912,002.

Graeme, B. (2007). Would you put a missioncritical network on wireless?. INSTRUMENTATION \& CONTROL.

Hasan, S. F.; Siddique, N. H. \& Chakraborty, S. (2012). Developments and constraints in 802.11-based roadside-to-vehicle communications. Wireless Personal Communications, 1-27.

Hu, Qi G. W., Williamson, C. \& Fapojuwo, A. O. (2011). Experimental evaluation of asymmetric QoS in IEEE $802.11 \mathrm{~g}$ wireless networks. En Proceedings of the 7th ACM symposium on QoS and security for wireless and mobile networks. ACM. 1-8.

Kisner, R. A., et al. (2010). Cybersecurity through real-time distributed control systems. Technical report, Oak Ridge National Laboratory (ORNL), Oak Ridge, Tennessee, USA.

Kritzinger, P. S., et al. (2010). Comparing the results from various performance models of IEEE 802.11 g DCF. Computer Networks, 54(10), 1672-1682.

Majkowski, J., Palacio, F. (2007). Calidad de Servicio en WLAN considerando un escenario mixto IEEE 802.11 e IEEE 802.11 b.

Malaney, R. (2006). Quality of Service Seeker. WIPO Patent No WO/2006/015427, 16. 
Mitra, B. et al. (2011). A Novel QoS Differentiation Framework for IEEE 802.11 WLANs: A Game-Theoretic Approach Using an Optimal Channel Access Scheme. Computational Intelligence and Information Technology, 500-502.

Mur, D. C., et al. (2010). On centralized schedulers for 802.11 e WLANs distribution versus grouping of resources allocation. Wireless Communications and Mobile Computing, 12.

Navajas, J. F. et al. (2005). Evaluación de QoS en accesos a Internet para aplicaciones Multimedia (EQoSIM). Proc. Mundo Internet, 2, 179-185.

Nieminen, J., Paloheimo, H., Jantti, R. (2010). Energy-adaptive scheduling and queue management in wireless LAN mesh networks. EnWireless Internet Conference (WICON), 2010 The 5th Annual ICST. IEEE, $1-9$.

Nwizege, K. S. (2011). Evaluation of Quality of Service (QoS) Support for Real-Time or Mission Critical Services over IEEE 802.11 e Wireless Networks. EnComputer Modeling and Simulation (EMS), 2011 Fifth UKSim European Symposium on. IEEE, 418-423.

Ong, E. H. \& Khan, J.Y. (2009). A unified QoS-inspired load optimization framework for multiple access points based wireless LANs. En Wireless Communications and Networking Conference, 2009. WCNC 2009. IEEE. IEEE. 1-6.

Panousopoulou, A. et al. (2004). Recent Trends on QoS for Wireless Networked Controlled Systems. University of Patras Electrical and Computer Engineering Department.

Paquette, A. (2011). Design of a Pragmatic Test Lab for Evaluating and Testing Wireless Medical Devices for Deployment on an Integrated IT Wireless Network.

Prasad N. R. \& Prasad A. R. (2004). 802.11 WLANs and IP Networking: Security, QoS, and Mobility. Prasad Anand.

Rappaport, T. S., et al. (2011). System, method, and apparatus for determining and using the position of wireless devices or infrastructure for wireless network enhancements. U.S. Patent No 8,019,352, 13.

Sarkar, M. \& Paolini, C. (2011). A QoS guaranteeing MAC layer protocol for the" underdog" traffic. EURASIP Journal on Wireless Communications and Networking, 2011(1), 1-15.

Seema, A. \& Reisslein, M. (1998). Towards efficient wireless video sensor networks: A survey of existing node architectures and proposal for a Flexi-WVSNP design. Communications Surveys \& Tutorials, IEEE, 13(3), 462-486.

Sen, S., Jawanda, J., Basu, K. \& Das, S. Quality-of-Service degradation strategies in multimedia wireless networks. Wireless Syst. Eng. Lab., Northern Telecom Inc., Richardson, TX; Vehicular Technology Conference. VTC 98. 48th IEEE. 
Seok-Kyu Kweon \& Kang G. Shin. (2003). Statistical Real-Time Communication over Ethernet.IEEE Transactions on Parallel and Distributed Systems, 14.

Serrano Y. P. (2006). Estrategias de Configuración de redes WLAN IEEE 802.11e EDCA. Universidad Carlos II de Madrid. Dep. de Ing. Telemática.

Shin-Jer Y. (2001). An approach to modeling performance evaluation on the ethernet with QoS parameters. International Journal of Network Management archive. 11(2), 91-101.

Simo R. J., et al. (2010). Modeling and optimizing IEEE 802.11 DCF for long-distance links. Mobile Computing, IEEE Transactions on, 2010, 9(6), 881-896.

Tim Szigeti \& Hattingh, Christina . (2004). End-to-End QoS Network Design. Cisco Press.

Tinnirello, I. \& Bianchi, G. (2010). Interference estimation in IEEE 802.11 networks. Control Systems, IEEE, 30(2), 30-43.

Ullah, I. (2012). A study and analysis of Public WiFi. Tesis Doctoral. Linköping.

Vassis, D., et al. (2010). Providing advanced remote medical treatment services through pervasive environments. Personal and Ubiquitous Computing, 14(6), 563-573.

Villegas, E., G., Ferré, R. V. \& Paradells, J. (2009). Frequency assignments in IEEE 802.11 WLANs with efficient spectrum sharing. Wireless Communications and Mobile Computing, 9(8), 1125-1140.

Weitzen, C. (2009). Service Differentiation Using Managed Sleep in CSMA/CA Networks. Tesis Doctoral. Wake Forest University.

Wentink, M. M., et al. (2012). Systems and methods for indicating buffered data at an access point using a traffic indication map broadcast. U.S. Patent No 8,089,908, 3.

Yang, S., Song W. \& Zhong, Z. (2012). Resource Allocation for Aggregate Multimedia and Healthcare Services over Heterogeneous Multi-Hop Wireless Networks. Wireless Personal Communications, 1-23.

Zhao, Y. (2012). Performance analysis for VoIP traffic with limited retransmissions in IEEE 802.11-based wireless networks. En Wireless Communications and Mobile Computing Conference (IWCMC), 2012 8th International. IEEE, 962-967.

Zhifeng T., S.S. (2004). Panwar. An analytical model for the IEEE 802.11e EDCF. Dept. of Electr. \& Comput. Eng., Polytech. Univ. Brooklyn, NY, USA. 